\title{
The AST3-NIR Camera for the Kunlun Infrared Sky Survey
}

\author{
Jon Lawrence*a Michael Ashley ${ }^{\mathrm{b}}$, Michael Burton ${ }^{\mathrm{b}}$, Xiangqun Cui ${ }^{\mathrm{c}}$, Vladimir Churilov ${ }^{\mathrm{a}}$, \\ Robert Content $^{\mathrm{a}}$, Peter Gillingham ${ }^{\mathrm{a}}$, Karl Glazebrook ${ }^{\mathrm{d}}$, Bozhong Gu ${ }^{\mathrm{c}}$, Michael Ireland $\mathrm{e}^{\mathrm{e}}$ \\ Xiang Jiang ${ }^{c}$, Haiping Lu ${ }^{c}$, Anna Moore ${ }^{f}$, Jeremy Mould ${ }^{d}$, Nicholas F. Staszak ${ }^{a}$, Julia Tims ${ }^{\mathrm{a}}$, \\ Peter Tuthill ${ }^{\mathrm{g}}$, Lifan Wang ${ }^{\mathrm{h}, \mathrm{i}}$, Xiangjan Yuan ${ }^{\mathrm{c}}$, Kaiyuan Zhang ${ }^{\mathrm{c}}$, Ross Zhelem ${ }^{\mathrm{a}}$, Jessica Zheng ${ }^{\mathrm{a}}$ \\ ${ }^{a}$ Australian Astronomical Observatory, North Ryde, NSW 2113, Australia; ${ }^{\mathrm{b}}$ School of Physics, \\ University of New South Wales, Sydney NSW 2052, Australia; ${ }^{\mathrm{c}}$ Nanjing Institute of Astronomical \\ Optics and Technology, National Observatories, Chinese Academy of Science, Nanjing 210042, \\ China; ${ }^{\mathrm{d}}$ Centre for Astrophysics and Supercomputing, Swinburne University of Technology, \\ Hawthorn, VIC 3122, Australia; ${ }^{\mathrm{e}}$ Research School for Astronomy \& Astrophysics, Australia \\ National University, Canberra ACT 2611, Australia; ${ }^{\mathrm{f}}$ Caltech Optical Observatories, California \\ Institute of Technology, Pasadena, CA 91125, USA; 'School of Physics, The University of Sydney,

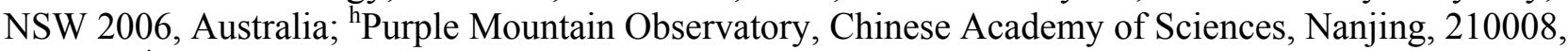 \\ China; ${ }^{\text {DDepartment }}$ of Physics, Texas A\&M University, College Station, TX 77843-4242, USA
}

\begin{abstract}
AST3-NIR is a new infrared camera for deployment with the AST3-3 wide-field survey telescope to Dome A on the Antarctic plateau. This project is designed to take advantage of the low Antarctic infrared sky thermal background (particularly within the $\mathrm{K}_{\text {dark }}$ near infrared atmospheric window at $2.4 \mu \mathrm{m}$ ) and the long Antarctic nights to provide high sensitivity temporal data from astronomical sources. The data collected from the Kunlun Infrared Sky Survey (KISS) will be used to conduct a range of astronomical science cases including the study of supernovae, exo-planets, variable stars, and the cosmic infrared background.
\end{abstract}

Keywords: Antarctic astronomy, infrared imaging, Dome A, AST3, PLATO, KISS

\section{INTRODUCTION}

It has long been recognized that the cold temperatures in the atmosphere above the Antarctic plateau should give rise to significant sensitivity gains for Antarctic observations in the infrared [1,2]. The latitude of the Antarctic continent also provides for a unique cadence for time series observations [3,4]. Calm atmospheric conditions also lead to high photometric precision [5]. The AST3-NIR project, currently in the preliminary design phase, is motivated to exploit these advantages. The facility will be used to conduct the Kunlun Infrared Sky Survey (KISS) from Dome A, with science goals addressing research across a range of galactic and extragalactic areas.

The baseline instrument concept for AST3-NIR consists of a $2.5 \mu \mathrm{m}$ cut-off HgCdTe detector array, mounted in a cryostat with relay optics, $\mathrm{K}_{\text {dark }}$ filter, internal baffling, a cold stop, and a focus drive. The optical relay provides a focalplane plate-scale that is optimised for high-sensitivity wide-field observations given the expected seeing conditions, the telescope diffraction limit, and the lens fabrication and alignment tolerances. The $\mathrm{K}_{\mathrm{dark}}$ filter bandwidth and centre wavelength is designed to maximise sensitivity for the expected atmospheric thermal emission spectrum and the telescope thermal emission properties. The cryostat design is driven towards reliability and simplicity by the extreme environmental conditions and the requirement for remote operation with only a yearly service interval.

The AST3-NIR camera mounts to a flange on the AST3-3 telescope tube. The telescope optical prescription is identical to the first two AST3 telescopes [6], which were designed for wide field of view optical application. The telescope is a modified Schmidt type with a $0.5 \mathrm{~m}$ plano-aspheric corrector plate followed by a $0.68 \mathrm{~m}$ oblate primary mirror with a flat folding secondary mirror. The telescope tube is enclosed and the corrector window has an indium-tin-oxide coating to avoid frost formation. The telescope and instrument include a control system that interfaces to a remote PLATO system $[7,8]$ for power generation, communications and control.

*j1@aao.gov.au; phone +61 2 9372-4853

Ground-based and Airborne Instrumentation for Astronomy VI, edited by Christopher J. Evans, Luc Simard, Hideki Takami Proc. of SPIE Vol. 9908, 990851 · (c) 2016 SPIE · CCC code: 0277-786X/16/\$18 · doi: 10.1117/12.2233317 


\section{SCIENCE GOALS}

The Kunlun Infrared Sky Survey's niche is in detection of changes in luminosity of astronomical sources in the infrared. This leads to a range of science goals that are described in detail by Burton et al. 2016 [9], which are aimed at providing a comprehensive exploration of the time varying Universe. The survey program under development has a number of key themes:

- Star formation: a survey to study dynamic interactions in the cores of stellar clusters, a study of the variability associated with accretion events in star clusters, and an examination of the intrinsic variability of young stellar objects;

- Dwarfs and planets: a dedicated survey searching for transiting Brown Dwarfs and Hot Jupiters with a potential secondary eclipse yielding key data on planet formation models, and a targeted search for transiting exoplanets around $\mathrm{M}$ dwarfs;

- Stellar evolution: a search for long-period variable stars in the Magellanic Clouds to unlock key aspects of the terminal phases of stellar evolution;

- Transients: a wide field survey with appropriate cadence is expected to discover and yield information and statistics on a wide range of transient events including fast radio bursts, gravitational wave sources, and gamma ray bursts.

- Supernovae: a search for a database of Type Ia supernovae to act as the low redshift anchor for the Hubble diagram where infrared observations have been used to improve systematics, and a search for dust-obscured supernovae buried in starburst galaxies;

- Active Galactic Nuclei: using reverberation mapping of AGN to characterize dust in the central disk or torus as a function of black hole mass and galaxy dynamics;

- The cosmic infrared background: a search for structure in the infrared background as a means to probe the epoch of reionisation.

\section{SYSTEM PARAMETERS}

The high level instrument parameters are given in Table 1. These parameter have been derived based on the science goals for the instrument and are discussed in more detail in the following sections.

Table 1. AST3-NIR system parameters.

\begin{tabular}{|l|l|}
\hline Parameter & Value \\
\hline Diameter of primary mirror & $0.68 \mathrm{~m}$ \\
\hline Effective diameter of primary mirror & $0.50 \mathrm{~m}$ \\
\hline System focal ratio (average) & $\mathrm{f} / 5.5$ \\
\hline Pixel size & $18 \mu \mathrm{m}$ \\
\hline Image scale & $1.35 \mathrm{"} /$ pixel \\
\hline Number of pixels & $2048 \times 2048$ \\
\hline Field of View & $46 \times 46$ arcmin \\
\hline Central wavelength & $2.375 \mu \mathrm{m}$ \\
\hline Bandwidth & $0.250 \mu \mathrm{m}$ \\
\hline
\end{tabular}




\section{TELESCOPE}

The Antarctic Survey Telescope-AST3 was designed to have three optical telescopes matched respectively with G, R, and I band filters. Each of the AST3 telescopes is a modified Schmidt type with an entrance pupil diameter of 500mm, a focal ratio of 3.73, and field of view of $4.14^{\circ}$. They consist of a transparent aspherical plate as the entrance pupil and an aspherical primary mirror with diameter $680 \mathrm{~mm}$. The first two AST3 telescopes [10] employ a spherical refractive corrector with a filter before the focal plane that uses a 10kx10k optical CCD array (STA1600-FT) with a plate scale of 1" per pixel. AST3-1 and AST3-2 were deployed to Dome A in early 2012 and early 2015 respectively [11,12].

Due to the great advantage of Dome A in the near infrared, the third AST3 telescope has been re-optimized for diffraction limited imaging in the $\mathrm{K}$ band. It uses the same entrance pupil and primary mirror as the first two AST3 telescopes since they were already manufactured at Nanjing Institute of Astronomical Optics and Technology (NIAOT). The AST3-NIR camera is interfaced to the main optical tube by adding in a fold mirror between the Schmidt corrector and the primary mirror - see Figure 1. The telescope system is designed with a single flange for operation only with the AST3-NIR K $\mathrm{K}_{\text {dark }}$ camera.

The baseline design to combat ice formation on the telescope optics is to use a sealed telescope tube with an indium-tinoxide (ITO) coating on the front corrector element. By applying a voltage across this film layer, the surface of the optical element and the nearby air can be heated to a few degrees above ambient. This has proven to be successful in several seasons of operation of the AST3-1 and AST3-2 telescopes. However the coating reduces the system transmission in the infrared and adds to the thermal background. For this reason, alternative techniques to avoid frost formation are currently being explored.

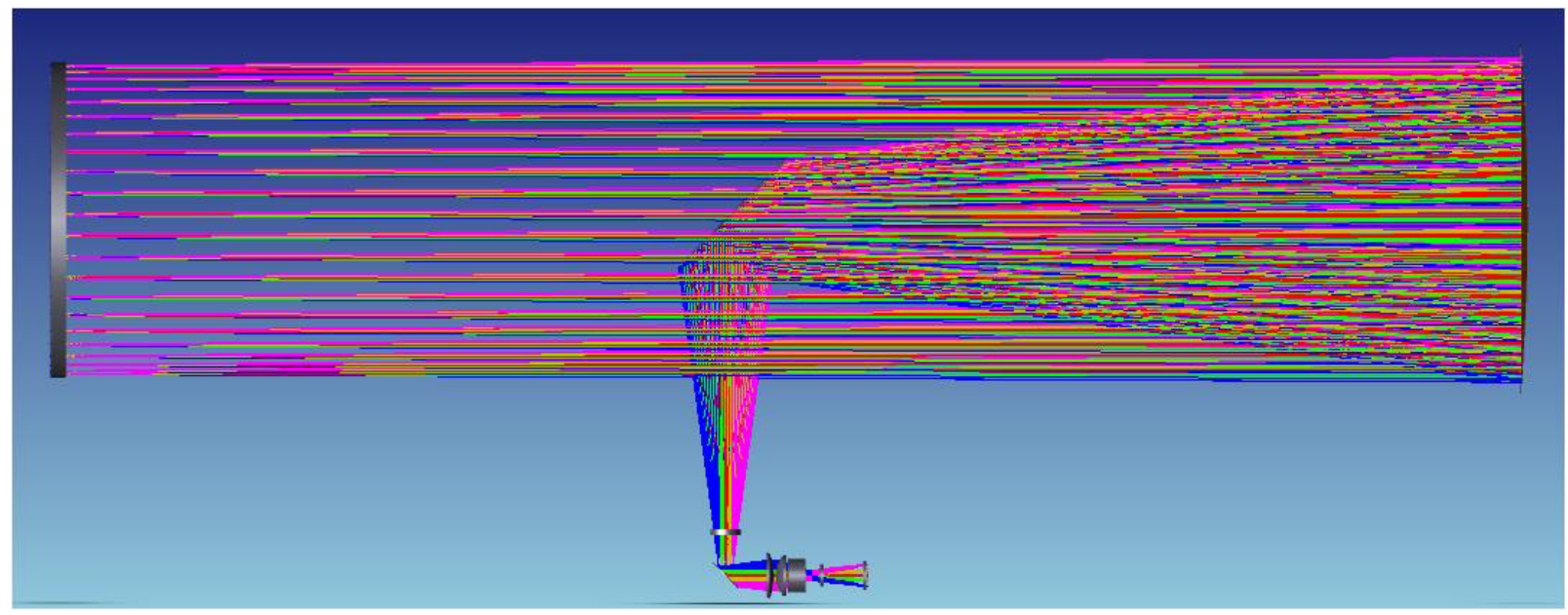

Figure 1. Optical layout for the AST3-3 telescope and camera.

\section{CAMERA}

\subsection{Operating wavelength and filter bandwidth}

An early design choice in this project, motivated by the desire to reduce costs and system complexity, was to consider only a single fixed wavelength for the camera. This obviates the need for a selectable filter system inside the instrument cryostat, which would have added to the cryostat size and added a requirement for a cryogenic mechanism.

With a single fixed filter, a considerable design task was to determine the optimum centre wavelength and bandwidth for this filter. The trade-off is described in detailed in Li et al. 2016 [13]. The selection was made based on a sensitivity calculator that incorporated a model of the key telescope and instrument parameters, a model of the atmospheric transmission spectrum, and a model of the atmospheric emission spectrum throughout the infrared $\mathrm{K}$ band. The atmospheric models were based on previous site testing data [1,14].

The sensitivity map based on this analysis is shown in Figure 2. The optimum wavelength $(2.375 \mu \mathrm{m})$ occurs close to the minimum in the atmospheric emission spectrum (bounded by $\mathrm{OH}$ emission blueward and thermal emission redward). 
The bandwidth selection $(0.25 \mu \mathrm{m})$ balances competing influence from the atmospheric transparency (for observations of bright sources) against the influence from the atmospheric emissivity (important for fainter sky-background limited observations).

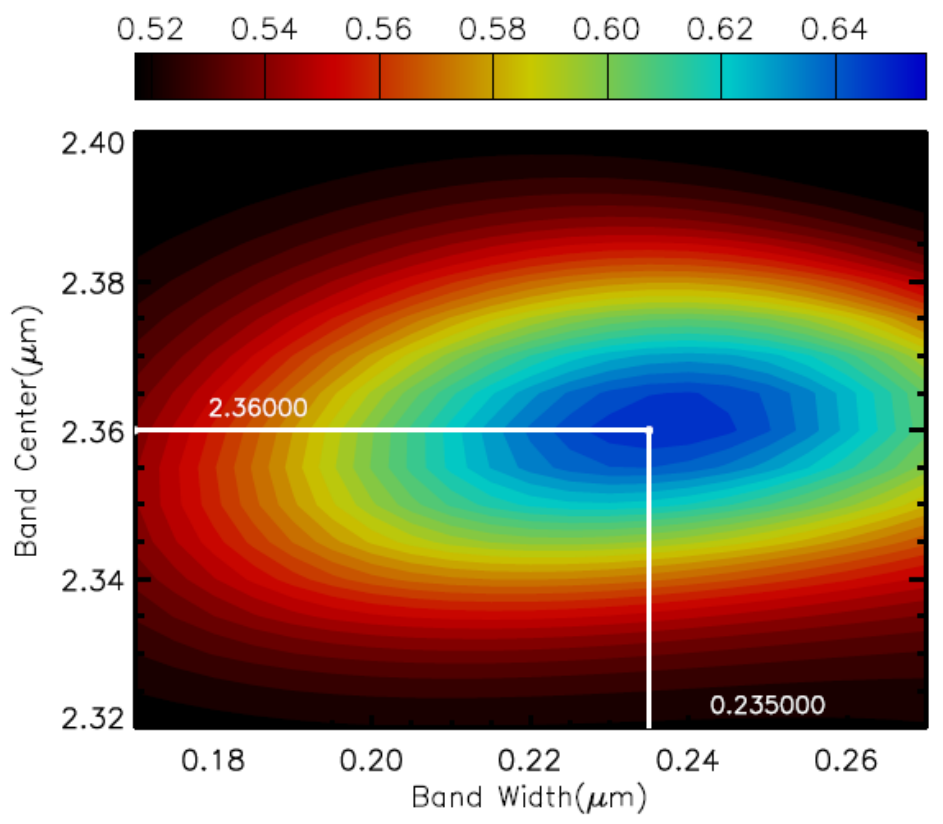

Figure 2. Sensitivity (in units of relative SNR) as a function of band centre and bandwidth (from [13]).

\subsection{Pixel scale}

The next design choice for the AST3-NIR camera was the pixel scale. This is driven by the science requirements that mostly involve wide field surveys. In this trade-off as the instrument field of view is increased the undersampling is also increased. There is a point at which increasing the field size does not bring gains in survey speed, because for background limited observations the sensitivity reduces with increased pixel size, although there is an additional overhead due to repointing.

The delivered image quality depends on an error budget with terms due to: diffraction, design image quality, static errors from tolerances and misalignments of the optical elements, dynamic errors from telescope tracking and/or wind shake, and atmospheric seeing. Diffraction from the $0.5 \mathrm{~m}$ aperture gives $\sim 1$ ", the optical design and tolerancing is assumed to add to $\sim 0.6 "$ image size, and the telescope errors are expected to be $<1 "$. There is no data on the seeing from Dome A available, but assumptions and extrapolations from other Antarctic plateau sites (South Pole [15], Dome C [16], and Dome F [17]) indicate the values should range from 0.2 " to $2.5 "$ at this wavelength. This drives us to a focal ratio giving a plate scale of 1.35"/pixel, representing $\sim 1$ pixel per FWHM in the best seeing conditions and $\sim 2$ pixels per FWHM in the worst seeing conditions, and a field-of-view of $46^{\prime} \times 46^{\prime}$.

\subsection{Thermal emission}

We considered two different approaches to ensure that the telescope thermal emission was not dominant. Our starting point was to design the simplest imaging system possible that comprised only a field flattening lens and a filter in the instrument cryostat close to the detector. This arrangement had no reimaged pupil plane and thus thermal emission from the telescope structure itself (mostly from within the telescope tube) would reach the detector unless it was blocked by a series of narcissus mirrors or cold baffles. The thermal emission model showed that this approach was problematic as even though ambient temperature at Dome A is low, telescope emission could easily dominate over atmospheric thermal emission without quite elaborate cold structures built into the telescope tube and narcissus mirrors that would block a significant fraction of the science beam. 
It was therefore decided to go with a more traditional approach to control thermal emission, i.e., to reimage the pupil plane onto a cold stop inside the cryostat. A thorough analysis of the thermal emission model (see Figure 3 for example), as described in [18], has shown that this solution is satisfactory.

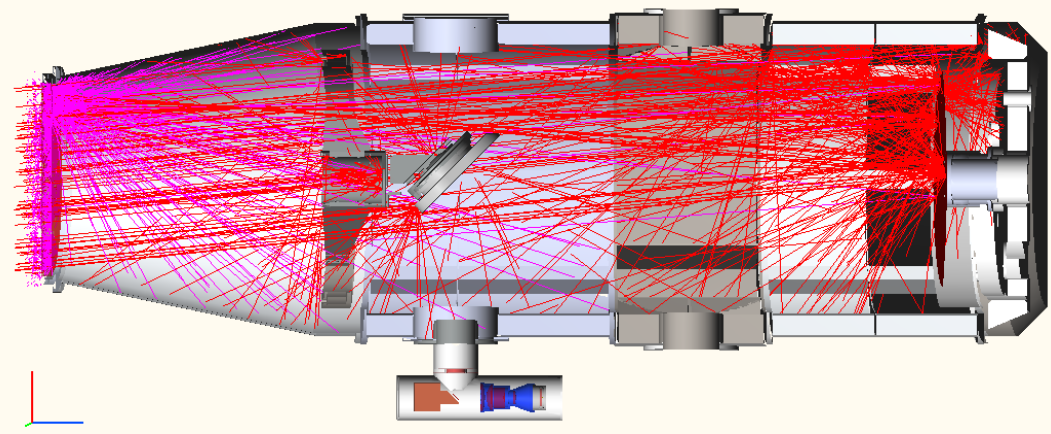

Figure 3. Analysis of scattered light within the telescope tube.

\subsection{Optical design}

The optical design flowed from the defined centre wavelength, the pixel scale requirement, the fixed optical prescription of the telescope front corrector and primary mirror, and the desire for a cold pupil. In addition, a constraint was added to have a flat cryostat window (to simplify alignment, assembly, and interfacing between the instrument and the telescope), and to have a folded light path (due to mechanical constraints). A series of 4 optical designs were developed and these were down-selected based on image quality and manufacturability of the lenses.

The selected design, shown in Figure 4, provides a magnifying focal plane relay giving an average system focal length of $\mathrm{f} / 5.5$ with an intermediate pupil plane. The optics comprise a flat fused silica cryostat window, followed by a flat fold mirror, followed by two ZnSe meniscus lenses, a fused silica meniscus with one aspheric surface, and a cold stop. Beyond the cold stop is a biconvex $\mathrm{ZnSe}$ lens, the filter, and the detector surface. Lens positions are relatively relaxed with typically 100-200 $\mu \mathrm{m}$ decenter and z-spacing allowed. Alignment of the camera with respect to the telescope is quite loose with $1 \mathrm{~mm}$ decenter and 1 degree tilt allowed.

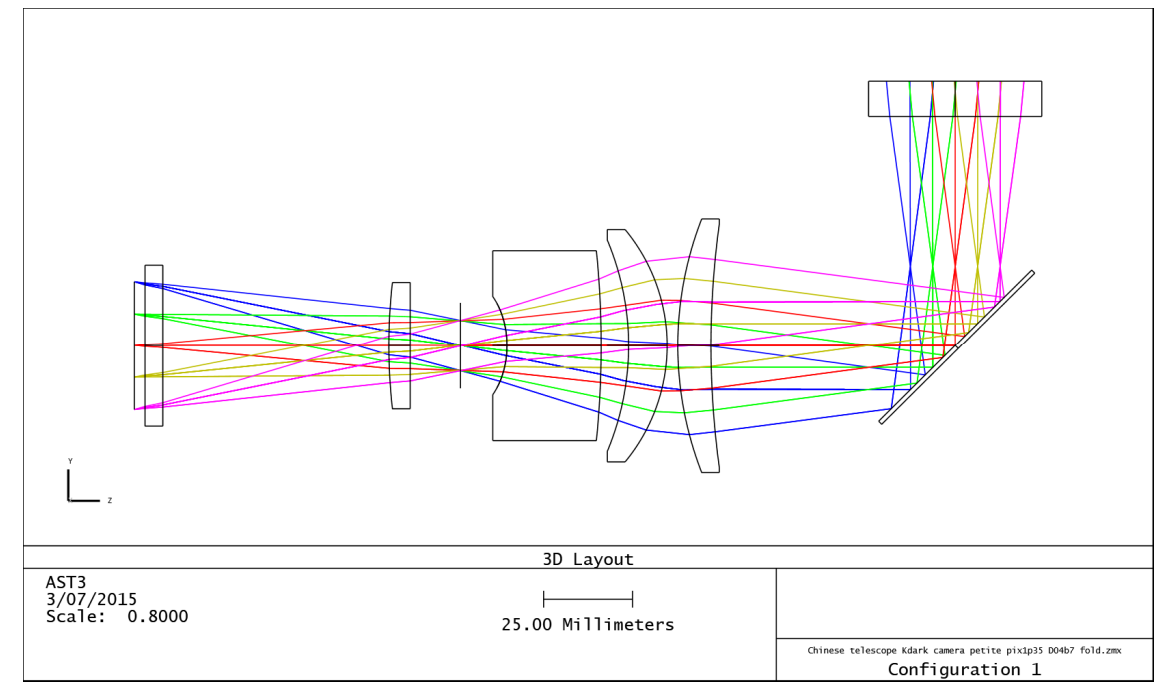

Figure 4. Optical layout for the AST3-NIR camera.

\subsection{Cryostat design}

The telescope interface is defined by the location of the internal fold mirror and mounting flange on the external telescope tube. This flange is designed to be readily accessed in the field. We use a folded layout to minimise momentum and ensure telescope stability and pointing. Figure 5 shows the location of the instrument mounted to the telescope. 

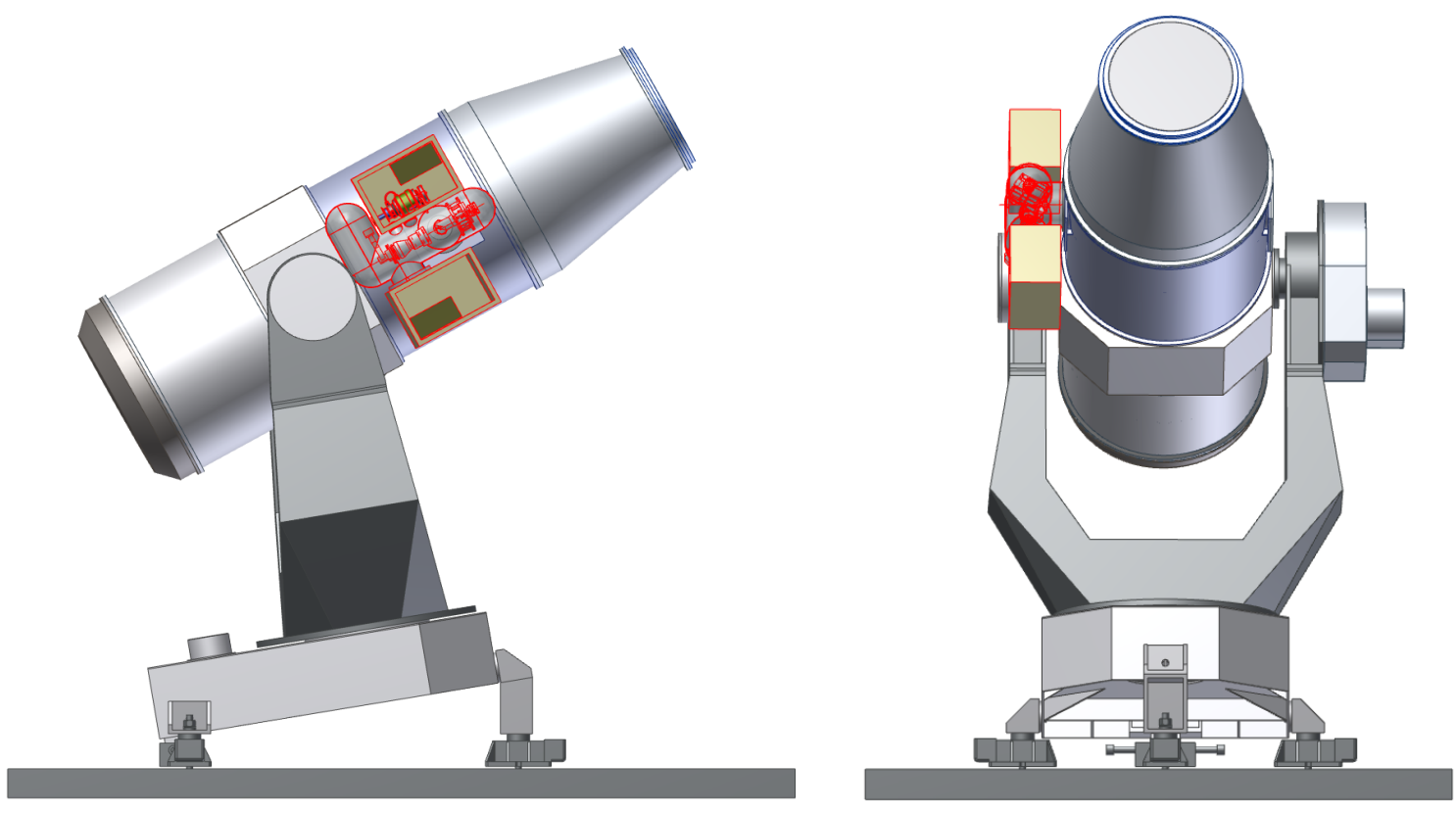

Figure 5. Instrument mounted to the side of the telescope tube.
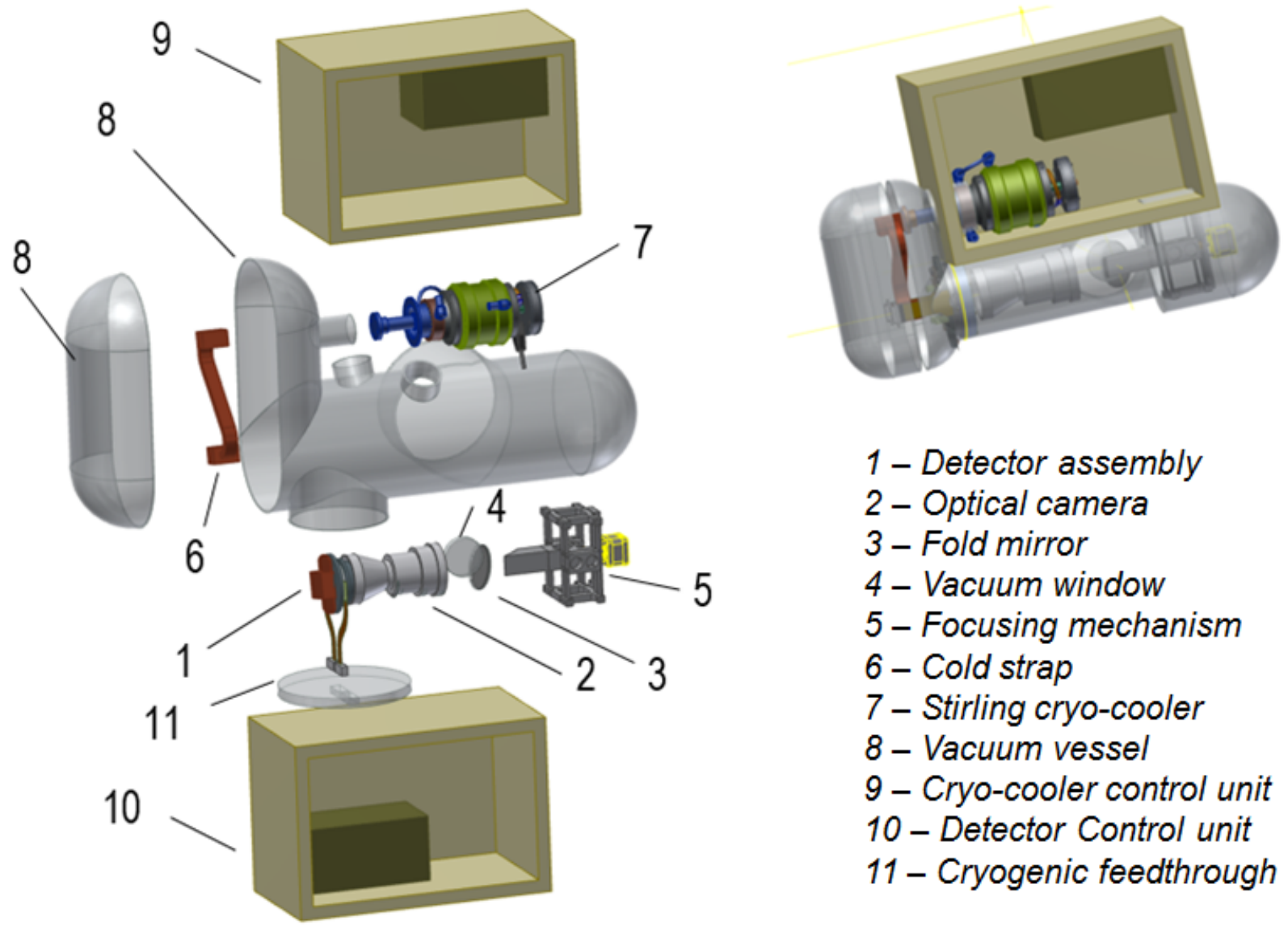

1 - Detector assembly

2 - Optical camera

3 - Fold mirror

4 - Vacuum window

5 - Focusing mechanism

6 - Cold strap

7 - Stirling cryo-cooler

8 - Vacuum vessel

9 - Cryo-cooler control unit

10 - Detector Control unit

11 - Cryogenic feedthrough

Figure 6. Concept design for the AST3-NIR camera. 
Key requirements driving the optomechanics and cryostat design include: a vacuum hold time of at least 12 months (i.e. greater than the service interval) with a goal of several years (to avoid re-pumping in the field); extreme environmental conditions (ambient temperature ranging from $183 \mathrm{~K}$ to $263 \mathrm{~K}$, atmospheric pressure $\sim 550$ mbar, and relative humidity $>100 \%$ ); working temperatures of $110 \mathrm{~K}$ for the detector and filter, $150 \mathrm{~K}$ for the optics and cold stop, and ambient for the cryostat window and fold mirror; fully remote operation; and limited facilities for in-field assembly, testing, and maintenance. These factors result in a need for a very high reliability system and hence a simple design that utilises successful technical solutions from previous instruments.

The mechanical concept (see Figure 6) uses a barrel with potted lens cells to hold the optics, and a focussing mechanism for the fold mirror with a design derived from a previous AAO cryogenic instrument [19]. The vacuum vessel is a compact design that folds around the camera optics tube, mirror focus mechanism, and detector assembly. The detector mounting assembly is made of molybdenum and connected via a cold strap to a SunPower Stirling cryo-cooler. This model of cryo-cooler has been proven to work in the harsh Antarctic environment [20]. Good thermal contract is established between the detector mounting assembly and the filter mount. A flexible PCB cable connects the detector to the pre-amp and external detector controller via a vacuum-rated high-density cryogenic feedthrough mounted on the cryostat Conflat flange. Metal gaskets are used for all off-the-shelf and in-house designed vacuum seals. External to the cryostat are thermally controlled enclosures that house the cryo-cooler electronics and the room temperature controller electronics.

\subsection{Detector and controller}

We are considering two options for the instrument detector and controller.

The baseline design uses a Hawaii 2RG device and integrated cryogenic controller from Teledyne. All deliverable elements are standard parts: a H2RG Science Grade HgCdTe $2048 \times 2048$ pixel array (see Figure 7), with cryogenic SIDECAR ASIC in a 337-pin ceramic package, cyrogenic PCB assembly with flex cable, external room-temperature acquisition module, and Teledyne SIDECAR ASIC Operating Software.

The second option being considered is from Selex ES. This option is based around a new array that was developed in 2014/15 by Selex ES, with a subcontract to UKATC for the controller, testing, and software aspects of the project. The deliverables (see Figure 7) would be: a HgCdTe $1276 \times 1024$ pixel array with ROIC multiplexer mounted on a standard test-card, an Astronomical Research Cameras (ARC) Leach Controller (electronics and housing), standard test interconnects, and UCam Operating software from UKATC.

Both Teledyne and Selex ES chips provide satisfactory performance: with QE $>0.7$ throughout the design bandwidth, dark current less than $1 \mathrm{e}^{-} / \mathrm{pixel} / \mathrm{sec}$ (this signal dominated by sky background), read noise $<5 \mathrm{e}^{-}$(Fowler) and $<25 \mathrm{e}^{-}$ (CDS), array read time of $<4$ secs, and power consumption $<100 \mathrm{~W}$. The main differentiator between the arrays in performance is pixel count, which is a strong driver for many of the wide-field science goals. There are also potential risks due to export licensing requirements, particularly for the Teledyne device.
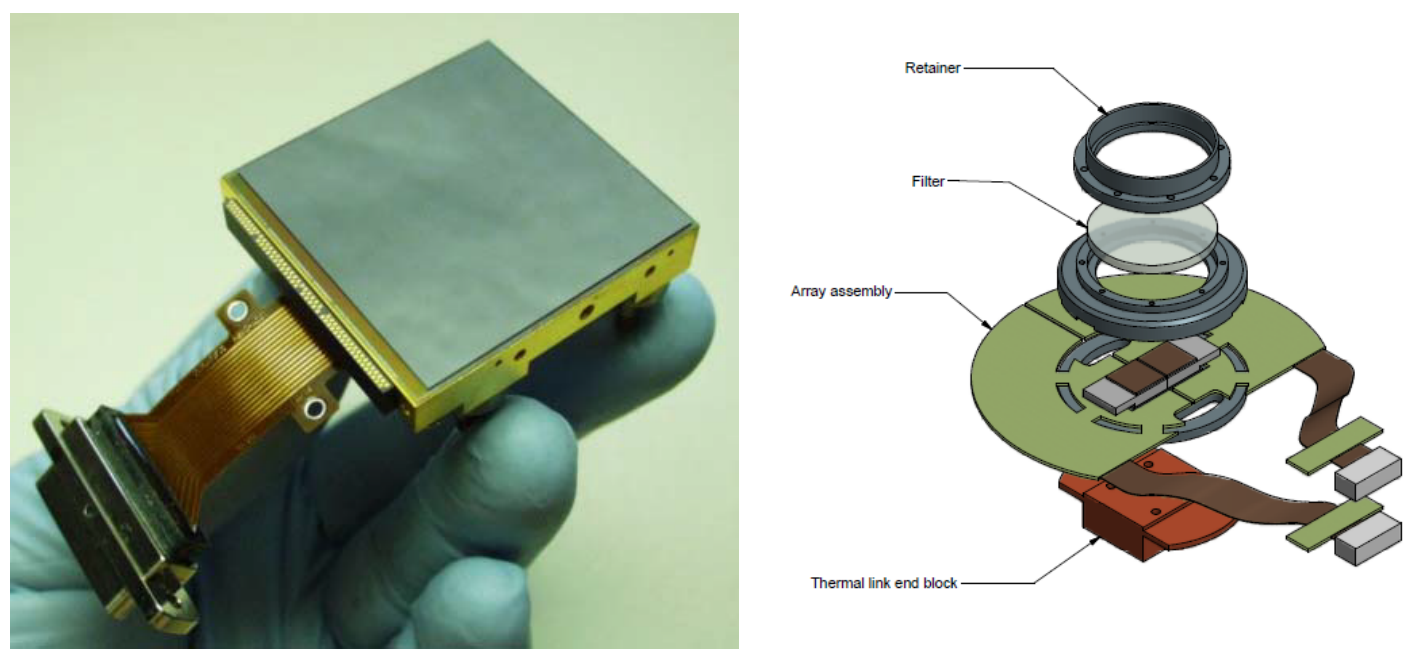

Figure 7. Left: picture of the Teledyne H2RG array; right: design interface for the Selex ES chip. 


\section{OPERATION AND DEPLOYMENT}

\subsection{Power and Communication}

The Kunlun station at Dome A is not manned over winter and typically only accessed (via overland traverse) for a few weeks in the summer. The KISS facility must therefore be designed for fully remote operation. As with the existing AST3 telescopes at Dome A, power, heat, and communications to the KISS facility will be provided by the PLATO (PLATeau Observatory) infrastructure that has operated reliably for over a decade in Antarctica [7,8]. The PLATO system uses diesel engines and photovoltaic panels to provide electrical power and heat (to a level up to $\sim 1 \mathrm{~kW}$ continuous), and uses the Iridium satellite network for communications (at a $128 \mathrm{kbps}$ bandwidth).

\subsection{Operation}

The KISS system in intended to operate similarly to the existing AST3 telescopes, with the instrument communicating via the PLATO module to a dedicated computer at the University of New South Wales, that can be accessed by members of the project consortium. The data collected by the AST3_NIR camera will be far too much to send via the limited bandwidth Iridium satellite link. Thus the full data-set must be stored on high-reliability hard-drives and physically collected at 1 year intervals (during the summer service mission). An automated data reduction pipeline will be developed that comprises scripts that can be run remotely for image construction, astrometry, and photometry.

\subsection{Assembly and Deployment}

There are a number of steps to the KISS facility development. The AST3-3 telescope is currently being assembled in China at NIAOT. Testing of the telescope is expected to occur over the next six months. In parallel with the telescope assembly, the AST3-NIR camera is being designed at the AAO. The camera procurement and assembly will continue for the next 12 to 18 months (with the detector procurement clearly the long lead time item that will drive the whole project schedule). After the camera is assembled and tested, the telescope will be shipped to Australia where system interfacing will occur, followed by full system tests. The instrument will then be removed from the telescope and both items shipped to Antarctica, where they will be reintegrated on-site at Dome A.

\section{SUMMARY}

Thanks to the low ambient temperature at Dome $\mathrm{A}$ and the low background sky radiation in the $\mathrm{K}_{\text {dark }}$ band, a telescope with a modest aperture and a relatively simple infrared camera can compete very strongly as a wide-field near-infrared survey instrument with existing mid-latitude telescopes. The KISS facility, which comprises the AST3-3 telescope and the AST3-NIR camera, will provide such a facility for the first time. KISS will be used to obtain a unique dataset leading to new science discovers of the time varying universe in the infrared. This project also provides the ideal opportunity for enhancing the Antarctic astronomy collaboration across institutes from Australia, China, and the United States, and for further developing the technology that will be vital to the success of larger scale Antarctic telescopes [21] and instruments in the future.

\section{REFERENCES}

[1] Ashley, M. C. B., Burton, M. G., Storey, J. W. V., Lloyd, J. P., Bally, J., Briggs, J. W., and Harper, D. A., "South Pole observations of the near-infrared sky brightness," Publ. Astron. Soc. Pac. 108, 721 (1996).

[2] Lawrence, J. S., "Infrared and sub-millimetre atmospheric characteristics of Antarctic high plateau sites," Publ. Astron. Soc. Pac. 116, 482 (2004).

[3] Wang, S., Zhang, H., Zhou, X., Zhou, J., Fu, J., Yang, M., Liu, H., Xie, J., Wang, L., Wang, L., Wittenmyer, R. A., Ashley, M. C. B., Feng, L., Gong, X., Lawrence, J. S., Liu, Q., Luong-Van, D. M., Ma, J., Peng, X., Storey, J. W. V., Wu, Z., Yan, J., Yang, H., Yang, J., Yuan, X., Zhang, T., Zhang, X., Zhu, Z., and Zou, H., "Photometic variability in the CSTAR field: results from the 2008 data set," Astrophys. J. Suppl. 218, 20 (2015).

[4] Huang, Z., Fu, J., Zong, W., Wang, L., Macri, L., Wang, L., Ashley, M. C. B., Cui, X., Feng, L., Gong, X., Lawrence, J., Liu, Q., Luong-Van, D., Pennypacker, C., Yang, H., Yuan, X., York, D. G., Zhou, X., Zhu, Z., and Zhu, Z., "Pulsations and period changes of the non-Blazhko RR Lyrae variable Y Oct observed from Dome A, Antarctica," Astron. J. 149, 25 (2015). 
[5] Kenyon, S. L., Lawrence, J. L., Ashley, M. C. B., Storey, J. W. V., Tokovinin, A., and Fossat. E., "Atmospheric Scintillation at Dome C, Antarctica: Implications for Photometry and Astrometry," Publ. Astron. Soc. Pac. 118, 924-932 (2006).

[6] Cui, X., Yuan, X., and Gong, X., "Antarctic Schmidt Telescopes (AST3) for Dome A," Proc. SPIE 7012, 70122D (2008).

[7] Lawrence, J. S., Ashley, M. C. B., Hengst, S., Luong-Van, D. M., Storey, J. W. V., Yang, H., Zhou, X., and Zhu, Z., "The PLATO Dome A site-testing observatory: Power generation and control systems," Rev. Sci. Instr. 80, 604501 (2009).

[8] Yang, H., Allen, G., Ashley, M. C. B., Bonner, C. S., Bradley, S., Cui, X., Everett, J. R., Feng, L., Gong, X., Hengst, S., Hu, J., Jiang, Z., Kulesa, C. A., Lawrence, J. S., Li, Y., Luong-van, D., Mccaughrean, M. J., Moore, A. M., Pennypacker, C., Qin, W., Riddle, R., Shang, Z., Storey, J. W. V., Sun, B., Suntzeff, N., Tothill, N. F. H., Travouillon, T., Walker, C. K., Wang, L., Yan, J., Yang, J., York, D., Yuan, X., Zhang, X., Zhang, Z., Zhou, X., and Zhu, Z., "The PLATO Dome A Site-Testing Observatory: Instrumentation and First Results," Publ. Astron. Soc. Pac. 121, 174-184 (2009).

[9] Burton, M. G., Zheng, J., Mould, J., Cooke, J., Ireland, M., Uddin, S. A., Zhang H., Yuan, X., Lawrence, J., Ashley, M. C. B., "Scientific goals of the Kunlun Infrared Sky Survey (KISS)," Publ. Astron. Soc. Aust., in press (2016).

[10] Yuan, X., and Su, D., "Optical system of the Three Antarctic Survey Telescopes," Mon. Not. R. Astron. Soc. 424, 23-30 (2012).

[11] Yuan, X., Cui, X., Gu, B., Yang, S., Du, F., Li, X., Wang, D., Li, X., Gong, X., Wen, H., Li, Z., Lu, H., Xu, ., Zhang, R., Zhang, Y., Wang, L., Shang, Z., Hu, Y., Ma, B., Liu, Q., and Wei, P., "The AST3 project: Antarctic Survey Telescopes for Dome A," Proc. SPIE 9145, 91450F (2014).

[12]Li, G., Fu, J., Liu, X., "Variable stars observed with the AST3-1 telescope from dome A of Antarctica," Research Astro. Astrophys., in press (2016).

[13]Li, Y., Zheng, J., Tuthill, P., Freeman, M., Ashley, M., Burton, M., Lawrence, J., Mould, J., and Wang, L., "Optimising the K Dark Filter for the Kunlun Infrared Sky Survey," Publ. Astron. Soc. Aust. 33, e008 (2016).

[14] Phillips, A., Burton, M. G., Ashley, M. C. B., Storey, J. W. V., Lloyd, J. P., Harper, D. A., and Bally, J., "The Near-Infrared Sky Emission at the South Pole in Winter," Astrophys. J. 527, 1009-1022 (1999).

[15] Travouillon, T., Ashley, M.C.B., Burton, M.G., Storey, J.W.V., and Loewenstein, R.F., "Atmospheric turbulence at the South Pole and its implications for astronomy," Astron. \& Astrophys. 400, 1163 (2003).

[16] Aristidi, E., Fossat, E., Agabi, A., Mékarnia, D., Jeanneaux, F., Bondoux, E., Challita, Z., Ziad, A., Vernin, J., and Trinquet, H., "Dome C site testing: surface layer, free atmosphere seeing, and isoplanatic angle statistics," Astron. Astrophys. 499, 955-965 (2009).

[17] Okita, H., Ichikawa, T., Ashley, M. C. B., Takato, N., and Motoyama, H., "Excellent daytime seeing at Dome Fuji on the Antarctic plateau," Astron. Astrophys. 554, L5 (2013).

[18]Zheng, J. R., Lawrence, J., Content, R., Churilov, V., Zhang, K., Yuan, X., and Lu, H., "Antarctic Survey Telescope (AST3-3) NIR camera for the Kunlun Infrared Sky Survey (KISS): Thermal Optimization and System performance," Proc. SPIE 9911, in press (2016).

[19] Tinney, C. G., Ryder, S. D., Ellis, S. C., Churilov, V., Dawson, J., Smith, G. A., Waller, L., Whittard, J. D., Haynes, R., Lankshear, A., Barton, J. R., Evans, C. J., Shortridge, K., Farrell, T., and Bailey, J., "IRIS2: a working infrared multi-object spectrograph and camera," Proc. SPIE 5492, 998-1009 (2004).

[20] Burton, M. G., Ashley, M. C. B., Braiding, C., Storey, J. W. V., Kulesa, C., Hollenbach, D. J., Wolfire, M., Glück, C., and Rowell, G., "The Carbon Inventory in a Quiescent, Filamentary Molecular Cloud in G328," Astrophys. J. 782, 72 (2014).

[21]Zhu, Y., Wang, L., Yuan, X., Gu, B., Li, X., Yang, S., Gong, X., Du, F., Qi, Y., and Xu, L., "Kunlun Dark Universe Survey Telescope," Proc. SPIE 9145, 91450E (2014). 